\title{
Lateralization of an auditory signal in correlated noise and in uncorrelated noise as a function of signal frequency*
}

\author{
DONALD E. ROBINSON \\ Indiana University, Bloomington, Indiana 47401 \\ and \\ JAMES P. EGAN \\ The University of Texas, Austin, Texas 78712
}

\begin{abstract}
Listeners lateralized a monaural signal presented against a continuous background of perfectly correlated noise (NO) or of uncorrelated noise (NU). Measures of signal detectability were also secured in separate tests. Psychometric functions (percent correct vs signal energy) were determined for each task. For a tonal signal of either low or high frequency, a listener requires only slightly greater signal energy (about $1 \mathrm{~dB}$ ) in order to lateralize as well as he can detect when the noise is uncorrelated (NU). When the noise is perfectly correlated (NO), the slope of the psychometric function for lateralization depends upon signal frequency. With $250 \mathrm{~Hz}$, the slope of the psychometric function for lateralization is much smaller than that for detection. With $1,000 \mathrm{~Hz}$, the function for lateralization is steeper than that for $250 \mathrm{~Hz}$, but the slope is still less than that of the function for detection for $1,000 \mathrm{~Hz}$. With $2,000 \mathrm{~Hz}$, the function for lateralization has about the same slope as that for detection.
\end{abstract}

In 1966, Egan and Benson reported on the ability of human listeners to lateralize a low-frequency tonal signal in a noise background. Their data indicate that when a $500 \cdot \mathrm{Hz}$ signal is presented monaurally $(\mathrm{Sm})$ in interaurally uncorrelated noise (NU) listeners show only a slight decrement in lateralization as compared to detection. However, when the noise has an interaural correlation of unity (NO), the psychometric function for lateralization is not only displaced toward greater signal energy compared to that for detection, but has a much lower slope.

Egan and Benson discuss their results in terms of the cues available to the listeners in the NO conditions. They point out that the interaural temporal difference which occurs at the signal frequency may either lead or lag in the ear that received the signal. This occurs because the signal is added at a random phase-angle, $\propto$, to the corresponding frequency component of the noise masker. Since this angle is rectangularly distributed, all possible values of the resulting interaural phase-angle, $\theta$, are equally probable, whether the signal is added at the left ear or at the right. The only other cue that is available for lateralization is some transform of the interaural level difference. Although a signal added to the noise in one ear may result in a decrease in level at that ear, on the average the waveform at the ear receiving the signal will have a higher level than will the waveform at the nonsignal ear. If it is assumed that the interaural time shift resulting from $\theta$ is the cue which produces the markedly greater detectability of NO-Sm compared to NU-Sm, the lack of this cue for

\footnotetext{
*The computer program which provided the best-fitting psychometric functions was written by William A. Yost. This research was supported in part by a contract from the Air Force Office of Scientific Research to James P. Egan.
}

lateralization may explain the resulting decrement in performance in lateralization. Egan and Benson explain the low slope of the NO-Sm lateralization function by suggesting that interaural temporal difference is affecting the lateralization task through a trading of time and intensity. Preliminary computations suggested to them that such a trading of time and intensity would lower the slope of the psychometric function of lateralization relative to that for detection. Another way of stating their argument is to say that the interaural temporal difference, which contains no information about which ear received the signal, acts as a confusor and leads to a decrement in performance in lateralization. Further, as signal level is increased, the magnitude of this temporal difference increases only slightly less rapidly than does the interaural level difference. As a result, the psychometric function for lateralization has a lower slope than does that for detection.

The preceding idea suggests that conditions which will reduce the magnitude of the available interaural temporal difference will increase the slope of the psychometric function for lateralization under NO-Sm. Consider the following example. The interaural temporal difference is a decreasing function of signal frequency. Thus, as the frequency of the signal is increased, the available interaural temporal difference will decrease, and for relatively high frequencies the auditory nervous system will be unable to encode the interaural temporal difference. When this occurs, the only cue available for lateralization will be the interaural intensitive difference, and the psychometric function for lateralization will have the same slope as that for detection.

\section{PROCEDURE}

The ability of a listener to lateralize a monaural signal was 


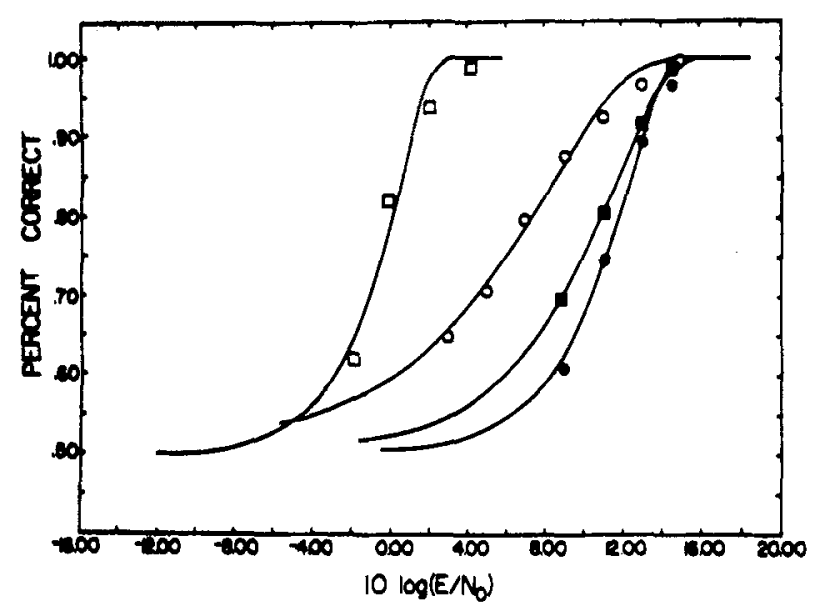

Fig. 1. Psychometric functions for $250 \mathrm{~Hz}$, for the four conditions: NO detection (a), NO lateralization (o), NU detection ( $\bullet$ ), and NU lateralization $(\bullet)$.

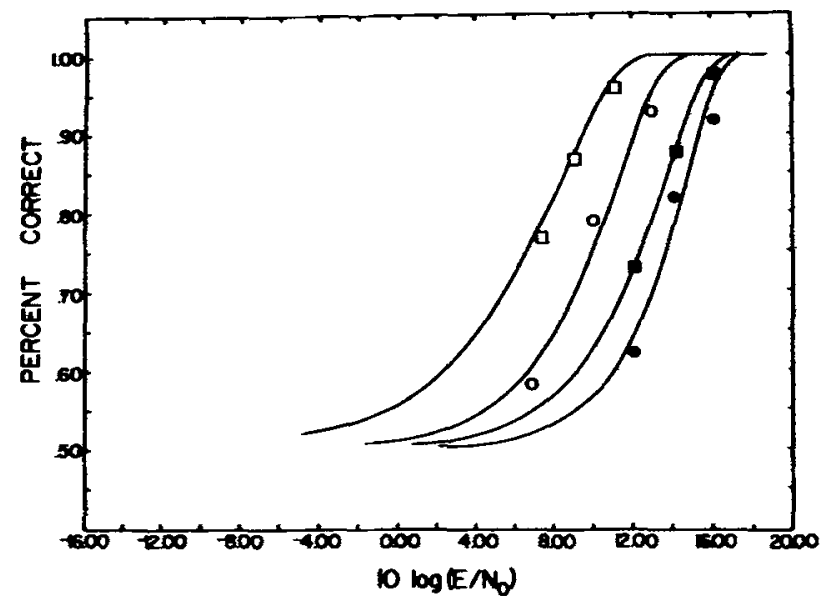

Fig. 2. Psychometric functions for $1,000 \mathrm{~Hz}$, for the four conditions: NO detection ( $($ ), NO lateralization (o), NU

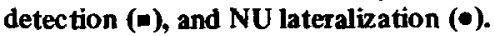

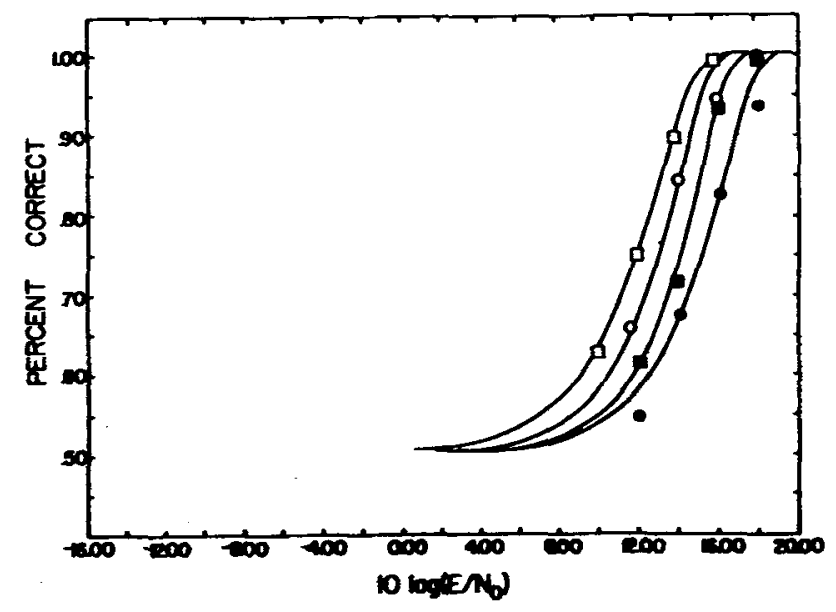

Fig. 3. Psychometric functions for $2,000 \mathrm{~Hz}$, for the four conditions: NO detection (o), NO lateralization $(\circ)$, NU

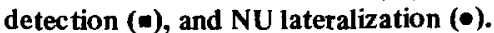

measured by the following procedure. ${ }^{1}$ A single observation interval was defined for the listener on each trial, and the monaural signal occurred during this observation interval on every trial. This signal was presented to either the right or the left ear by random determination, $p(R)=p(L)$, and the listener was instructed to indicate which ear received the signal. Each trial consisted of the following temporal sequence: a warning light $(0.5 \mathrm{sec})$, pause $(0.5 \mathrm{sec})$, light for observation interval $(0.25 \mathrm{sec})$, response interval $(2.0 \mathrm{sec})$, feedback light $(0.5 \mathrm{sec})$.

Measures of signal detection were secured under conditions similar to those used for the tests of lateralization. The method called "two-alternative, temporal forced choice," or $2 \mathrm{~A}, \mathrm{TFC}$, was employed for the measurement of signal detection. The monaural signal was presented on every trial, and it occurred with equal probability in either the first or the second of two observation intervals. The two lights which defined the two observation intervals were spatially discrete, and these two intervals were separated by a pause of $0.5 \mathrm{sec}$. Otherwise, the sequence of events for each trial was the same as that described for the task of lateralization. Although the monaural signal was presented to the same ear throughout a block of trials, each ear was tested in order to determine whether the signal had the same detectability for one ear as for the other.

All test units consisted of blocks of 80 trials. Four to six blocks were conducted during each session. After preliminary training, frequent "tone demonstrations" and practice blocks at high signal levels were conducted during each session.

The listening crew consisted of four young women; they were paid for their services for the 43 sessions. One listener did not complete the tests. All four listeners had clinically normal audiograms.

For all tests, the signal was a tone burst with a duration of $250 \mathrm{msec}$. The first series of tests was conducted with a tone of $250 \mathrm{~Hz}$, the second series with $2,000 \mathrm{~Hz}$, and the third with $1,000 \mathrm{~Hz}$. The tone was turned on without regard to phase and without the use of special devices so that the (negligible) transients were determined by the response of the earphones (Permoflux Corporation, PDR-10). The noise was band-limited only by the earphones and therefore had a nearly uniform spectrum level from about 100 to about $7,000 \mathrm{~Hz}$, and it was generated by a 6D4 tube (noise generators, Model 455-B, Grason-Stadler Company). The spectrum level of the noise was $45 \mathrm{~dB}$ re 0.0002 microbar in each ear for all tests, and it was present continuously throughout each block of trials. The members of a listening crew were tested simultaneously, and the input voltages were the same for all crew members. The energy of the signal, $E$, was varied from one block of trials to the next so that the percentage of correct decisions could be plotted against $10 \log \left(E_{s} / N_{0}\right)$.

At each frequency of the signal, the two tasks of lateralization of detection were conducted under each of two binaural conditions of masking. Two noise generators, one for each channel, were used to obtain the binaural condition called "uncorrelated noise," NU, and one noise generator was used to obtain the condition called "perfectly correlated noise,". NO.

\section{RESULTS}

Psychometric functions based on the averages of the data from the three Os are shown in Figs. 1-3. The functions for detection show the percentage of trials on which the Os identified correctly which of the two, temporally discrete, observation intervals contained the signal. Since the data showed negligible differences between ears for the detection tasks, the psychometric functions for detection are based upon the average of the data from the right and left ears. The psychometric functions for lateralization show the percentage of trials 
on which the Os identified correctly the ear to which the monaural signal was delivered.

Each of the psychometric functions in Figs. 1-3 is based on a minimum chi square fit to the function $\mathrm{d}^{\prime}=$ $m\left(E_{s} / N_{o}\right)^{k}$. The data points shown in the figures are the average percent correct for the three Os. Functions for each $\mathrm{O}$ are not shown since it is felt that the average data are representative of those from each of the Os.

The slope (or steepness) of a psychometric function is measured by the value of $k$ in the best fitting equation: small values of $k$ indicate that $P(C)$ increase slowly with increasing values of $10 \log \left(\mathrm{E}_{\mathrm{s}} / \mathrm{N}_{\mathrm{O}}\right)$; large values indicate a more rapid change in $P(C)$ with increases in $10 \log \left(E_{s} / N_{o}\right)$. The values of $k$ for the data in Figs. 1-3 are shown in Table 1 . The psychometric functions obtained in the present experiments indicate that (a) for the detection conditions, there is no systematic change in $\mathrm{k}$ as a function of frequency of correlated noise (NO), (b) for the lateralization task and uncorrelated noise (NU), $\mathrm{k}$ is an increasing function of frequency. The effect of frequency on $k$ for uncorrelated noise and the task of detection is ambiguous. Two of the three Os show strictly monotonically increasing values of $k$ with increasing frequency for the NU detection task. The functions based on average data also show this result. However, the slope changes are small, relative to the slopes, and, therefore, the result is questionable. (A small change in $\mathrm{k}$ represents a large change in slope when $\mathrm{k}$ is small, but a smaller change when $\mathrm{k}$ is large.)

The position of a psychometric function along the abscissa is measured by the fitting parameter, $m$. The following observations apply to the individual psychometric functions as well as to those based on the average data: (a) For Condition NO and the tasks of detection and lateralization, the higher the signal frequency, the greater is the value of $10 \log \left(E_{s} / N_{o}\right)$ required for any particular level of performance; (b) for $\mathrm{NO}$, an increase in signal frequency affects the detection task more than it does the lateralization task; and (c) for $\mathrm{NU}$, a change in signal frequency has about the same effect on the functions for detection as it does on those for lateralization. The present data may also be viewed in terms of the difference in signal energy required for the same level of performance in the two tasks: detection and lateralization. If such a comparison is made at $P(C)=0.75$, it is found that for all three frequencies and for both noise conditions, lateralization requires more signal energy than does detection. For Condition NU, the lateralization task requires about $1 \mathrm{~dB}$ more signal than does the detection task at each of the three frequencies. For the NO condition, however, the decrement is a function of frequency. At $250 \mathrm{~Hz}$, about $6 \mathrm{~dB}$ more signal is required for lateralization than for detection, while at $1,000 \mathrm{~Hz}$, the decrement is about $3 \mathrm{~dB}$, and at $2,000 \mathrm{~Hz}$, it is about $1 \mathrm{~dB}$.

\section{DISCUSSION}

The model described in the introduction is similar to
Table 1

Slopes (Values of k) for Average Psychometric Functions for Both Correlated Noise (NO) and Uncorrelated Noise (NU) for the Two Tasks and the Three Frequencies

\begin{tabular}{clcccc}
\hline \multirow{2}{*}{ Condition } & & \multicolumn{3}{c}{ Frequency } \\
\cline { 5 - 7 } \cline { 5 - 6 } Noise & \multicolumn{1}{c}{ Task } & & $250 \mathrm{~Hz}$ & $1000 \mathrm{~Hz}$ & $2000 \mathrm{~Hz}$ \\
\multirow{2}{*}{ NO } & Detection & & 1.8 & 1.0 & 1.4 \\
& Lateralization & & 0.8 & 1.3 & 1.6 \\
\multirow{2}{*}{ NU } & Detection & & 1.0 & 1.3 & 1.9 \\
& Lateralization & & 1.5 & 1.6 & 1.5 \\
\hline
\end{tabular}

that suggested by Hafter (1971). The model proposes that the lateralization judgment is made on the basis of the sign of the quantity $\Delta:$ the weighted sum of the interaural temporal difference, $\Delta t$, and the interaural intensive difference in decibels, $\Delta \mathrm{I}$. The model is thus stated $\Delta=\Delta \mathrm{t}+\Delta \mathrm{I} \cdot \mathrm{TR}$, where TR is the trading ratio in microseconds per decibel. ${ }^{2}$

Hafter, in his application of the model, assumed a rectangular distribution on the angle of addition of signal to noise, $\alpha$; a constant noise amplitude, $\mathrm{N}$; and computed an average of the absolute values of $\Delta$. In the present application, the probability of obtaining a positive value of $\Delta$ was computed assuming a rectangular distribution for $\alpha$ and a Rayliegh distribution for the noise amplitude. All other assumptions and computational techniques were similar to those employed by Hafter. The probability of obtaining a positive value of $\Delta$ was equated with the probability of a correct response in the lateralization task. Then psychometric functions for lateralization were obtained for various values of TR and signal frequency.

The theoretical psychometric functions, like those obtained from the Os, show increasing slope with increasing signal frequency. However, it was impossible to obtain adequate fits of the theoretical functions to the empirical ones. The theoretical functions, even for extremely large values of TR, are considerably less steep than the empirical ones. For example, at $250 \mathrm{~Hz}$, the Os showed a slope $(\mathrm{k})$ of 0.8 for lateralization, while the model yielded a $k$ of 0.39 with a TR of 40 microsec and a k of 0.50 with a TR of 100 microsec. At $2,000 \mathrm{~Hz}$, the $\mathrm{k}$ for the Os was 1.6, while the model produced $\mathrm{ks}$ of 0.55 and 0.54 for TRs of 40 and 100. If it is assumed that the $\mathrm{O}$ makes his judgment on the basis of $\Delta \mathrm{I}$ only, the predicted psychometric functions are still too shallow to fit the data. Several possibilities exist to explain the failure of the model to predict the details of the data. One of these is that the trading relation itself may be a function of frequency. Although this suggestion is plausible in the light of data from lateralization experiments, it is hardly useful here, because the present experiment does not allow an independent estimate of the trading relation or of its rate of change with frequency. A second possibility, also supported by lateralization data, is that the trading relation is a function of signal level. Since the MLD is decreasing with increasing frequency, the signal levels 
employed are increasing. Again, the experiment does not allow an estimate of this effect. It is also possible that the addition of "noise" on the decision axis would have improved the fit of the theoretical functions to the empirical ones. (See the discussion by Hafter, 1971, p. 1112.) In the absence of information about the form of the distribution of such a noise, however, it was felt that such an attempt would be premature. An obvious additional possibility is that the model is incorrect.

In general, we may conclude that interaural temporal differences act to degrade lateralization in noise, that lateralization is determined by a combination of interaural temporal and intensive differences, and that increasing signal frequency alters the shape of the psychometric function for lateralization by decreasing the magnitude of the temporal cue. However, we must also conclude that the particular form of the function relating lateralization performance in noise to signal frequency is not understood.

\section{REFERENCES}

Egan, J. P., \& Benson, W. Lateralization of a weak signal presented with correlated and with uncorrelated noise. presented with correlated and with uncorrelated noise. 20-26.

Hafter, E. R. Quantitative evaluation of a lateralization model of masking-level differences. Journal of the Acoustical Society of America, 1971, 50, 1116-1122.

Yost. W. A. Tone-on-tone masking for three binaural listening conditions. Journal of the Acoustical Society of America, $1072,52,1234-1237$.

\section{NOTES}

1. The two procedures used in the present study were nearly identical to those used for the Principal Experiments of Egan and Benson (1966), and much of this section is taken from their paper.

2. This model is similar to a model described by Yost (1872) and applied by him to tone-on-tone masking.

(Received for publication July 6, 1973; revision received October 30, 1973.) 